Supporting Material

\title{
Quintet State Electron Spin Resonance Spectra of Pyridyldinitrenes
}

Sergei V. Chapyshev, ${ }^{a, *}$ Richard Walton, ${ }^{b}$ Paul R. Serwinski, ${ }^{b}$ Paul M. Lahti, ${ }^{\text {b,* }}$

${ }^{a}$ Institute for Chemical Physics, Chernogolovka, RUSSIA

${ }^{\mathrm{b}}$ Department of Chemistry, University of Massachusetts, Amherst, MA 01003 USA 
Summary of optimized geometrical coordinates and spin density population analysis from Spartan 2002 for 2,4-pyridyldinitrene. UB3LYP/6-31G* optimization of the $\mathrm{S}=2$ state.

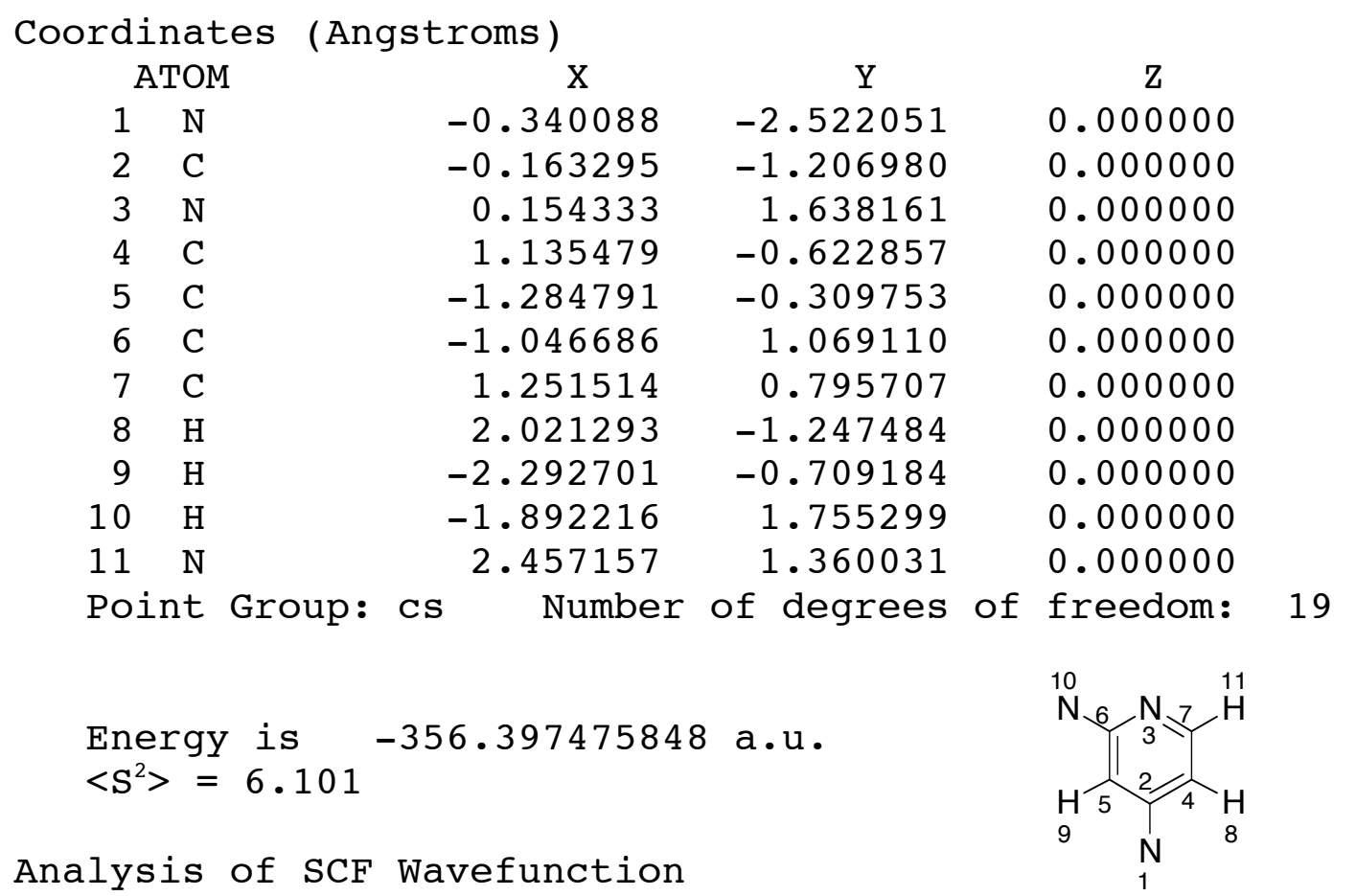

Mulliken Net Atomic Charges

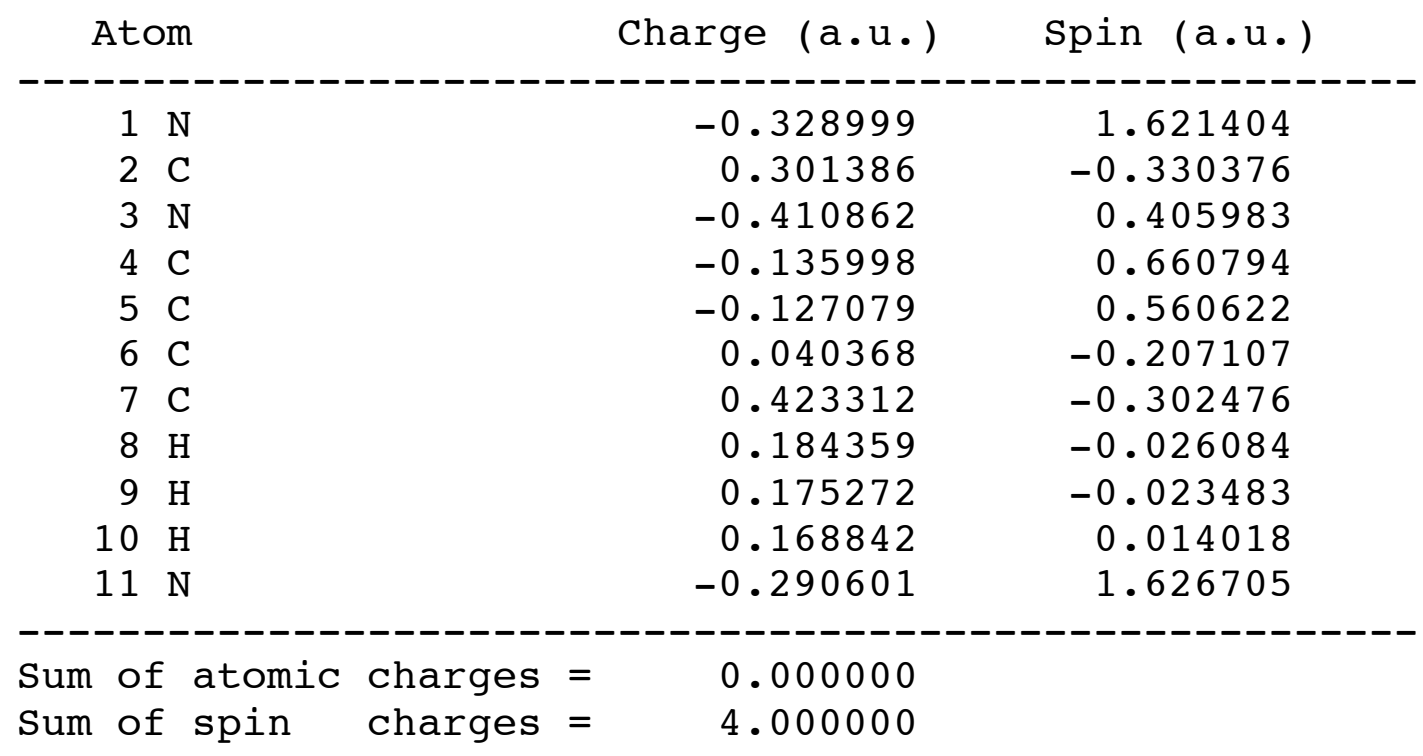


Summary of optimized geometrical coordinates and spin density population analysis from Spartan 2002 for 2,6-pyridyldinitrene. UB3LYP/6-31G* optimization of the $\mathrm{S}=2$ state.

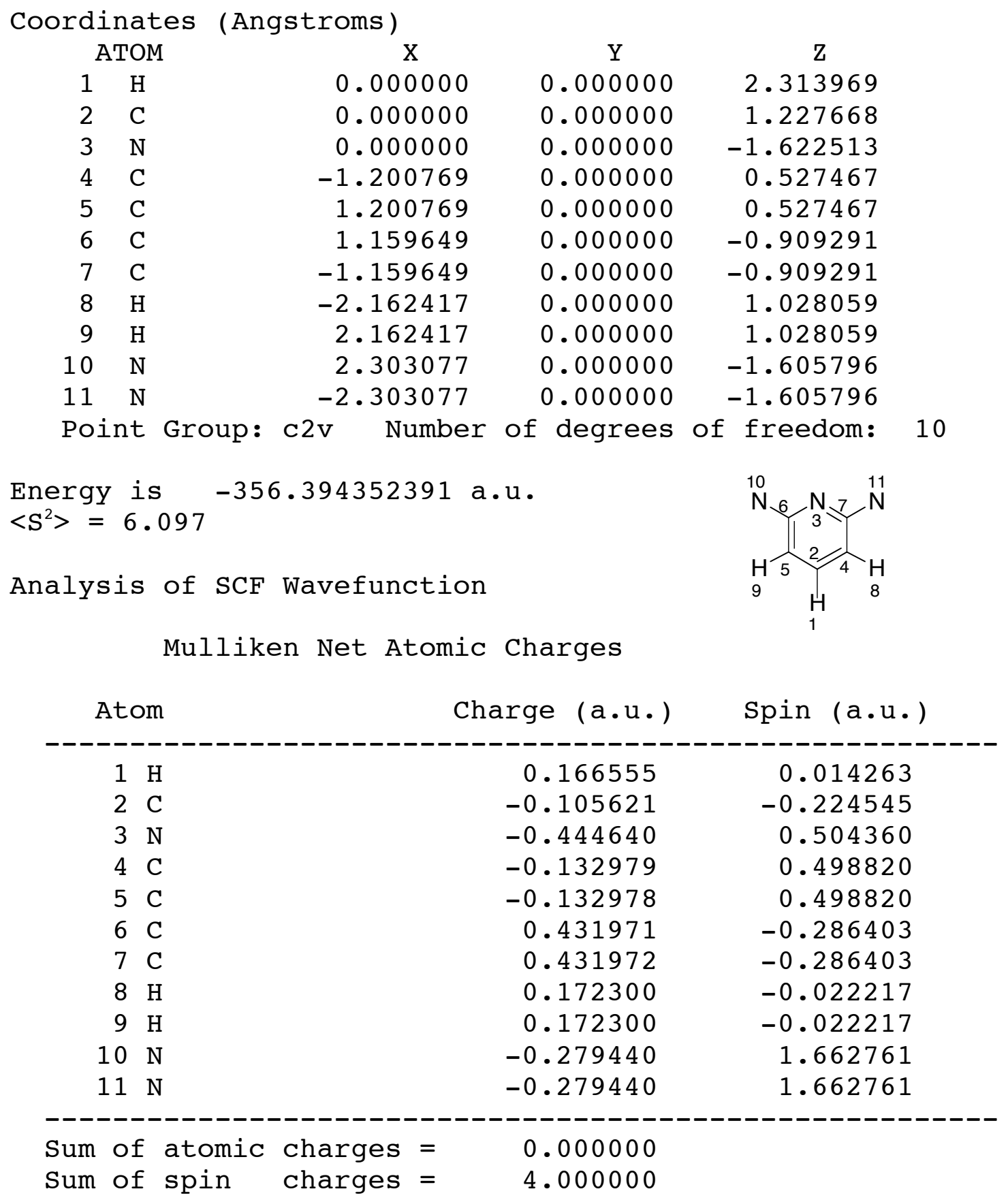


Summary of optimized geometrical coordinates and spin density population analysis from Spartan 2002 for 1,3-phenylenedinitrene. UB3LYP/6-31G* optimization of the $\mathrm{S}=2$ state.

\begin{tabular}{rrrrr}
$\begin{array}{c}\text { Coordinates } \\
\text { ATOM }\end{array}$ & (Angstroms) & \multicolumn{1}{l}{ X } & \\
1 & $\mathrm{H}$ & 0.000000 & 0.000000 & 2.512859 \\
2 & $\mathrm{C}$ & 0.000000 & 0.000000 & 1.426236 \\
3 & $\mathrm{C}$ & 0.000000 & 0.000000 & -1.404212 \\
4 & $\mathrm{C}$ & -1.213925 & 0.000000 & 0.746195 \\
5 & $\mathrm{C}$ & 1.213925 & 0.000000 & 0.746195 \\
6 & $\mathrm{C}$ & 1.239203 & 0.000000 & -0.696001 \\
7 & $\mathrm{C}$ & -1.239203 & 0.000000 & -0.696001 \\
8 & $\mathrm{H}$ & -2.159309 & 0.000000 & 1.277355 \\
9 & $\mathrm{H}$ & 2.159309 & 0.000000 & 1.277355 \\
10 & $\mathrm{~N}$ & 2.390622 & 0.000000 & -1.350826 \\
11 & $\mathrm{~N}$ & -2.390622 & 0.000000 & -1.350826 \\
12 & $\mathrm{H}$ & 0.000000 & 0.000000 & -2.488327 \\
Point Group: $\mathrm{C} 2 \mathrm{v}$ & Number of degrees of freedom: & 11
\end{tabular}

$$
\begin{aligned}
& \text { Energy is }-340.368966597 \mathrm{a} \cdot \mathrm{u} . \\
& \left\langle\mathrm{S}^{2}\right\rangle=6.102
\end{aligned}
$$

Analysis of SCF Wavefunction

Mulliken Net Atomic Charges

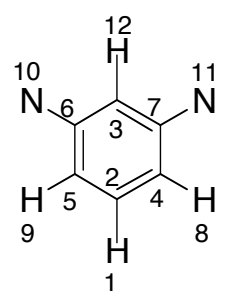

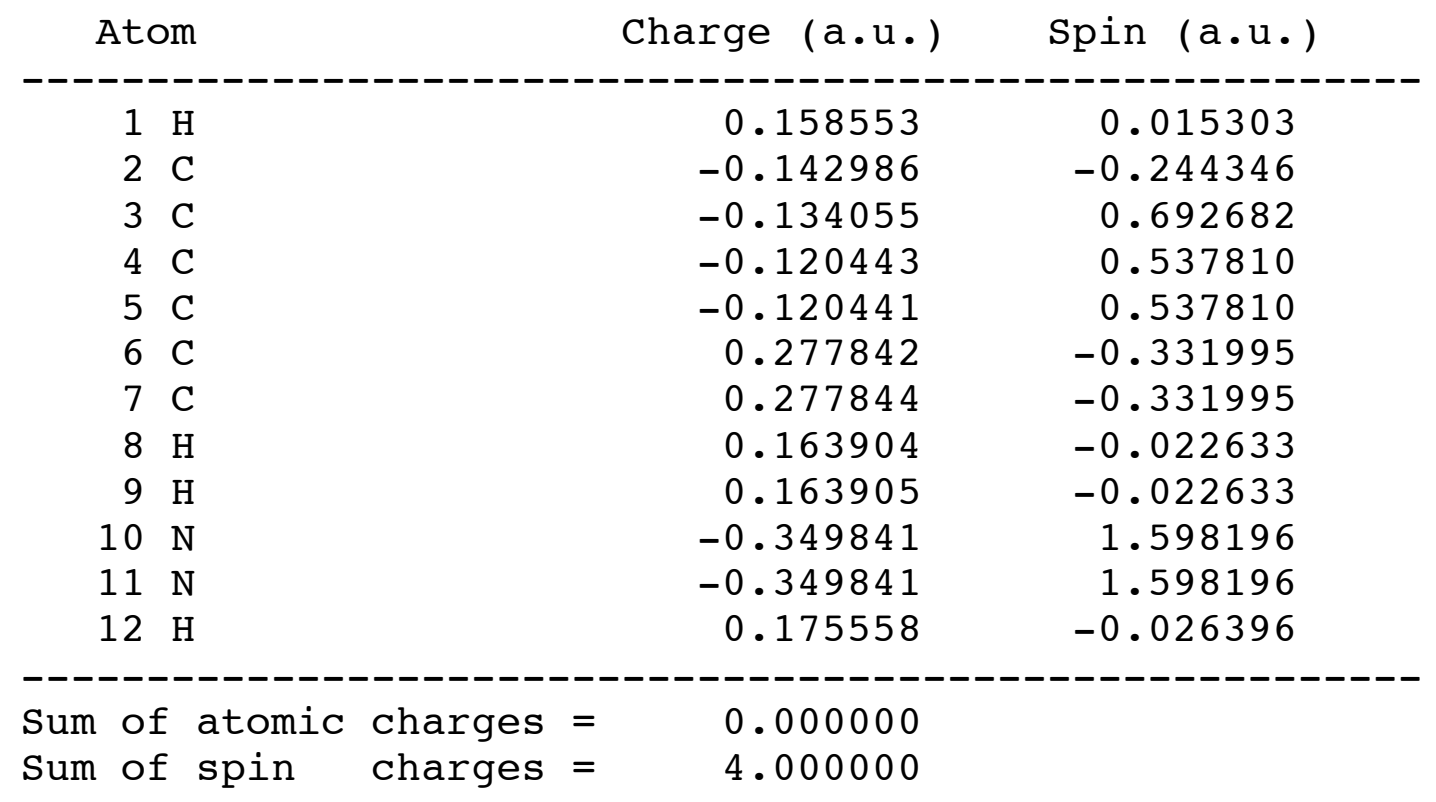

